\title{
Correspondence
}

\section{Space Medicine: Why Do Recently Published Papers about Telomere Length Alterations Increase our Uncertainty Rather than Reduce it?}

\author{
Bevelacqua J. J. ${ }^{10}$, Welsh J.2, Mortazavi S. A. R. ${ }^{3}$, Keshavarz \\ M. ${ }^{4}$, Mortazavi S. M. J. ${ }^{5 *(0)}$
}

\begin{abstract}
There is a growing interest in examining alterations in telomere length as a reliable biomarker of general health, as well as a marker for predicting later morbidity and mortality. Substantial evidence shows that telomere length is associated with aging; telomere shortening acts as a "counting mechanism" that drives replicative senescence by limiting the mitotic potential of normal (but not malignant) cells. In this Correspondence, we attempt to answer the question of why recently published papers about telomere length alterations increase our uncertainty rather than reduce it. This discussion includes three major research areas regarding telomere length: environmental stressors, aging, and life span. Our review suggests that activation of telomerase activity due to stressors in space might be a double-edged sword with both favorable and unfavorable consequences. The selection of an effect's consequence must clearly elucidate the experimental conditions as well as associated stressors. In this Correspondence, we attempt to answer the question of why recently published papers about telomere length alterations increase our uncertainty rather than reduce it. The selection of an effect's consequence must clearly elucidate the experimental conditions as well as associated stressors. Both positive and negative consequences must be clearly addressed in order to bolster the conclusions, as well as identify future research directions.
\end{abstract}

Citation: Bevelacqua JJ, Welsh J, Mortazavi SAR, Keshavarz M, Mortazavi SMJ. Space Medicine: Why Do Recently Published Papers about Telomere Length Alterations Increase our Uncertainty Rather than Reduce it? J Biomed Phys Eng. 2021;11(1):103-108. doi: 10.31661/jbpe.v0i0.2005-1115,

\section{Keywords}

Telomere; Aging; Lifespan; Stressors; Environment; Adaptive Response; Radiation; Space

\section{Correspondence,}

With Telomeres, the specialized DNA protective structures (caps) on the ends of chromosomes, protect the integrity of information-carrying DNA during successive cellular divisions by preventing base pair loss of chromosomal DNA. In other words, telomeres help prevent genome instability. Telomere shortening that occurs as a result of cellular replication over time, decreases the telomere length until the telomere becomes too short for the cell to divide, leading to a permanent cell cycle arrest known as replicative senescence $[1,2]$. Telomeres are normally shortened after each cellular division but in some cells their length is restored by telomerase, an RNA-containing reverse transcriptase enzyme (human telomerase reverse transcriptase or hTERT). Telomere maintenance plays a key role in continuous proliferation of cells that
${ }^{1} \mathrm{PhD}$, Bevelacqua

Resources, Richland,

Washington 99352,

United States

${ }^{2} \mathrm{MD}$, PhD, Loyola Uni-

versity Chicago, Edward

Hines Jr., VA Hospi-

tal, Stritch School of

Medicine, Department

of Radiation Oncology,

Maywood, IL 60153 USA

${ }^{3} \mathrm{MD}$, School of Medi-

cine, Shiraz University

of Medical Sciences,

Shiraz, Iran

${ }^{4} \mathrm{MSc}$, Department of

Medical Physics and

Engineering, School of

Medicine, Shiraz Univer-

sity of Medical Sciences,

Shiraz, Iran

${ }^{5} \mathrm{PhD}$, Department of

Medical Physics and

Engineering, School of

Medicine, Shiraz Univer-

sity of Medical Sciences,

Shiraz, Iran

*Corresponding author:

S. M. J. Mortazavi

Department of Medical

Physics and Engineering,

School of Medicine, Shi-

raz University of Medical

Sciences, Shiraz, Iran

E-mail: mortazavismj@ gmail.com

Received: 17 May 2020 Accepted: 27 July 2020 
are mitotically active [3]. Today, there is a growing interest in examining alterations in telomere length as a reliable biomarker of general health, as well as a marker for predicting later morbidity and mortality [4]. Substantial evidence shows that telomere length is associated with aging; telomere shortening acts as a "counting mechanism" that drives replicative senescence by limiting the mitotic potential of normal (but not malignant) cells. Moreover, an association between constitutive telomere length and cancer as well as several other disorders is shown in epidemiological studies [5]. It has even been reported that the individual differences in telomere length can be linked to individual differences in human behavior [6]. However, contribution of telomere length to neurological disorders still remains controversial [7]. Short telomeres can be linked to cardiovascular disease, at least in part through insulin-mediated pathways [8]. Besides genetic factors, some studies show that environmental stressors can also affect telomere length during growth and development [5]. Danese and Lippi in their report entitled "Telomere length: is the future in our "ends"?" state that "Albeit it is now undeniable that our future is largely in our "hands" (i.e., genotype, diet, exposure to environmental factors and so forth), larger and more solid evidence will be necessary before concluding that the future is also written in our (chromosome) "ends"."'[9]. A study conducted on 113 clean-up workers of Chornobyl nuclear power plant (NPP) accident showed a trend towards reducing the relative telomere length in clean-up workers who suffer from COPD and exposed to doses ranged 100 to $500 \mathrm{mSv}$ and above $500 \mathrm{mSv}$. In this study, the dose in clean-up workers ranged from 1.0 to $880 \mathrm{mSv}$ (330.4 $\pm 317.7(\mathrm{M} \pm \mathrm{SD}))[10]$.

In this Correspondence, we try to answer the question of why recently published papers about telomeres length alterations increase our uncertainty rather than reduce it. Our discussion includes three major fields regarding telomere length: environmental stressors, aging, and life span.

\section{A. Telomere Length and Space Envi- ronmental Stressors}

Garrett-Bakelman et al., in their paper "The NASA Twins Study: A multidimensional analysis of a year-long human spaceflight" published recently in Science [11] addressed the physiological, telomeric, transcriptomic, epigenetic, proteomic, metabolomic, immune, microbiomic, cardiovascular, vision-related, and cognitive effects of a 340-day mission onboard the International Space Station (ISS) in a male astronaut (Scott Kelly) compared to those of his monozygotic twin (Mark Kelly). This study showed that the majority of the biological and human health variables remained stable, or returned to baseline, after a yearlong human spaceflight. However, persistence of certain molecular changes (e.g., gene expression) should be taken into account for planning future longer missions ( $>1$ year).

Despite its undeniable strengths, the authors have not properly addressed the following issues:

1. The authors highlighted the effects of metabolic and nutritional status, physical activity, and weight loss on telomere length of the astronauts. However, space radiation (particularly high atomic number, high energy (HZE) particles) play an important role in telomere length changes. A key question about the elongation of Scott Kelly's telomeres during space flight is whether we can interpret it as a positive (adaptive) response to multiple changes in the environment (e.g. higher levels of radiation, microgravity, and spacecraft electromagnetic fields and atmosphere)? The issue of adaptive response in space mission and its importance as an efficient method of biological protection dates back to 2003 [12]. Different aspects of this issue are well-discussed in our recent publications $[13,14]$. At first glance, it can be hypothesized that this response is entirely a protective adaptive response (e.g. akin to constriction of our pupils when we move 
from a dim place to a much brighter environment). However, although stem and progenitor cells express telomerase, telomerase expression is generally silent in most somatic cells. In contrast, the elongation of telomeres by telomerase activity is a phenomenon which makes malignant cells immortal and is a hallmark of cancer [15]. In fact, although some cancer cells do not express telomerase, cell line immortality and telomerase activity is observed in about $90 \%$ of human cancer cell lines. Some studies suggest that all humans harbor malignant cells, but as long as they have an active immune system, these cells do not proliferate uncontrolably and there is no overt clinical cancer. This can be considered the "equilibrium" phase of the immunoediting hypothesis [16]. Moreover, telomerase inhibition has been introduced as a promising strategy for cancer treatment. Therefore, activation of telomerase activity due to stressors in space might be a double-edged sword with both favorable and unfavorable consequences.

2. The NASA Twin study findings might show subtle difference between exposure to low- and high-LET radiation. While neither the residents of high background radiation areas (HBRAs) of Ramsar, Iran [17] nor Kerala, India [18] exhibit telomere length alterations, the Twin Study clearly did document a significant change. It is worth noting that the shortcomings of the Ramsar study have already been addressed by Bevelacqua et al, [19]. While both space and certain HBRAs expose people to high-LET radiation, the different impacts on telomere lengths imply that other factors are also at work that we might not understand or have complete awareness. Different energy spectra of the particles, as well as the presence of heavy ions such as iron, oxygen, and carbon may also be involved in this difference.

3. Translocations are among several stable chromosome aberrations which persist many years after exposure. However, the paper by Garrett-Bakelman et al. [11] shows that the frequency of translocations decreased postflight either in HR (i.e., the Flight Subject) or TW (i.e., the Ground Subject). Inversions are another form of stable chromosomal aberrations and post-flight decreased inversion in HR twin is not justified. These changes may be due to large variations of the collected data. Given this consideration, these findings should be interpreted with caution.

4. The NASA conclusions are based on a 340-day mission to the ISS. Drawing definitive conclusions regarding longer duration space missions (e.g., a nominal $920 \mathrm{~d}$ Mars mission) must recognize that this difference affects the total delivered dose to the astronauts. Even if the background radiation from galactic cosmic radiation (GCR) and solar particle event (SPE) source terms remains constant, the difference in radiation shielding characteristics of the ISS and Mars mission vehicle [20] add additional differences that require consideration before extrapolating any ISS radiobiological conclusions to longer duration space missions. Moreover, the ISS is somewhat protected by the Earth's geomagnetic field, but this shielding is insignificant during the space mission outside the low-Earth orbit environment. In addition, the Martian magnetic field is significantly smaller in magnitude than Earth's geomagnetic field.

\section{B. Telomere Length and Aging}

Muñoz-Lorente et al., in their paper entitled "Mice with hyper-long telomeres show less metabolic aging and longer lifespans" that was published in Nature Communications Journal [21] reported that hyper-long telomere mice showed less DNA damage with aging. Moreover, these laboratory animals were lean and had lower levels of cholesterol and LDL, improved tolerance of glucose and insulin as well as less incidence of cancer and an increased longevity. Given these considerations, Muñoz-Lorente et al., concluded that not only longer than normal telomeres in a given species are not deleterious, but they are linked to 
some beneficial effects.

Welsh et al., have recently reviewed the findings of "The NASA Twins Study" that addressed the biological effects including telomere length changes of a 340-day mission onboard the International Space Station (ISS) in Scott Kelly, a male NASA astronaut compared to those in his monozygotic twin [22-24]. They also reviewed recent findings regarding the lack of telomere length alterations in the residents of high background radiation areas of Ramsar, Iran [17] and Kerala, India [18]. In their studies, Welsh et al., came to this conclusion that at least in some situations, elongation of telomeres is only a natural, protective adaptive response. Furthermore, as telomerase activity is a hallmark of cancer which grants immortality to malignant cells, they concluded that telomerase activity along with immune system dysregulation can increase the risk of cancer. Therefore, the reader of the paper authored by Miguel A. Muñoz-Lorente et al., should be aware of this key point that telomere elongation can be a response to a wide variety of stressors including radiation and microgravity. In particular, this commentary illustrates that a basic omission of Ref. 1 is the potential negative effects of telomere elongation. We believe that a better understanding of this "other side of the coin" is required for a better evaluation of the importance of telomere length alterations.

\section{Telomere Length and Life Span}

Arbeev et al., in their paper entitled "Association of Leukocyte Telomere Length With Mortality Among Adult Participants in 3 Longitudinal Studies" that was published in JAMA Network Open [25] attempted to answer the question whether leukocyte telomere length is associated with the natural life span of contemporary humans? This cohort study included 3259 adults of European ancestry. The results of their study showed that Leukocyte telomere length (LTL) was associated with a natural life span limit in contemporary humans. Despite its strengths, this paper has some shortcomings as follows:

1. The NASA twin study showed that telomere length is dynamic and can be changed as a simple response to environmental stressors. Given this consideration, the authors should have studied the occupational and residential stressors (e.g. exposure to UV, radon, and genotoxic chemical agents).

2. Studies performed on the residents of HBRAs either in Ramsar or Kerala shows that a low-level stressor such as elevated natural radiation doesn't cause telomere length changes. However, multiple stressors or high level stressors, such as those faced by astronauts in a deep space mission, can change the telomere length.

3. In the paper published in JAMA, it's puzzling that telomere length only affected noncancer mortality. This is exactly what the authors claim "Leukocyte telomere length-associated mortality from noncancer causes increased as participants aged, approaching their age at death". However, the paper by GarrettBakelman [11] shows that at age 50, telomere length can increase the Hazard Ratio of cancer from 0.8 to 1.2. This paper reveals that at ages 60, 70 and 80 cancer risk increased exponentially.

Another omission comes from ignoring key factors other than telomere length that affect lifespan. In 2005 Halaschek-Wiener et al., reported that they successfully identified longevity-associated genes in a long-lived Caenorhabditis elegans daf-2 (insulin/IGF receptor) mutant. Their study showed that reduction of daf-2 signaling in daf-2 mutant worms could double the mean lifespan [26]. More recently, Zullo et al., in a paper published in Nature, reported a conserved mechanism of ageing that is mediated by neural circuit activity and regulated by a protein called REST, which controls the expression of numerous genes involved in neural firing [27].

This Correspondence illustrates the impor- 
tance of considering all aspects of a presumed observation and its consequences. In the case of telomere length changes, both positive and negative effects are possible. The selection of an effect's consequence must clearly elucidate the experimental conditions as well as associated stressors. Both positive and negative consequences must be clearly addressed in order to properly characterize the conclusions as well as identify future investigations.

\section{Conflict of Interest}

None

\section{References}

1. Arsenis NC, You T, Ogawa EF, Tinsley GM, Zuo L. Physical activity and telomere length: Impact of aging and potential mechanisms of action. Oncotarget. 2017;8(27):45008-19. doi: 10.18632/oncotarget.16726. PubMed PMID: 28410238. PubMed PMCID: PMC5546536.

2. Victorelli S, Passos JF. Telomeres and cell senescence-size matters not. EBioMedicine. 2017;21:1420. doi: 10.1016/j.ebiom.2017.03.027.

3. Sobinoff AP, Pickett HA. Mechanisms that drive telomere maintenance and recombination in human cancers. Current Opinion in Genetics \& Development. 2020;60:25-30. doi: 10.1016/j. gde.2020.02.006.

4. Enlow MB, Kane-Grade F, De Vivo I, Petty CR, Nelson CA. Patterns of change in telomere length over the first three years of life in healthy children. Psychoneuroendocrinology. 2020;115:104602. doi: 10.1016/j.psyneuen.2020.104602.

5. Srinivas N, Rachakonda S, Kumar R. Telomeres and Telomere Length: A General Overview. Cancers. 2020;12(3):558. doi: 10.3390/cancers12030558.

6. Bateson M, Nettle D. Why are there associations between telomere length and behaviour? Philos Trans R Soc Lond B Biol Sci. 2018;373(1741):20160438. doi: 10.1098/rstb.2016.0438. PubMed PMID: 29335363. PubMed PMCID: PMC5784059.

7. Thanseem I, Viswambharan V, Poovathinal SA, Anitha $A$. Is telomere length a biomarker of neurological disorders? Biomarkers in Medicine. 2017;11(9):799-810. doi: 10.2217/bmm-20170032.

8. Zhan Y, Hagg S. Telomere length and cardiovascular disease risk. Curr Opin Cardiol. 2019;34(3):2704. doi: $10.1097 / \mathrm{HCO} .0000000000000613$.
9. Danese $\mathrm{E}$, Lippi G. Telomere length: is the future in our "ends"? Ann Transl Med. 2018;6(13):280. doi: 10.21037/atm.2018.06.24. PubMed PMID: 30094266. PubMed PMCID: PMC6064787.

10. Shvaiko LI, Bazyka KD, Sushko VO, Ilienko IM, Bazyka DA. Lung Function and Telomere Relative Length in Clean-Up Workers of Chornobyl Npp Accident in a Remote Post-Accident Period. Probl Radiac Med Radiobiol. 2019;24:503-15. doi: 10.33145/2304-8336-2019-24-503-515. PubMed PMID: 31841491.

11. Garrett-Bakelman FE, Darshi M, Green SJ, Gur RC, Lin L, et al. The NASA Twins Study: A multidimensional analysis of a year-long human spaceflight. Science. 2019;364(6436):eaau8650. doi: 10.1126/ science.aau8650.

12. Mortazavi SMJ, Cameron JR, Niroomand-Rad A. Adaptive response studies may help choose astronauts for long-term space travel. Advances in Space Research. 2003;31(6):1543-51. doi: 10.1016/S0273-1177(03)00089-9.

13. Bevelacqua JJ, Mortazavi SMJ. Commentary: human pathophysiological adaptations to the space environment. Frontiers in Physiology. 2018;8:1116. doi: 10.3389/fphys.2017.01116.

14. Bevelacqua JJ, Welsh J, Mortazavi SMJ. Comments on 'An overview of space medicine'. British Journal of Anaesthesia. 2018;120(4):874-6. doi: 10.1016/j.bja.2017.12.015.

15. Hanahan D, Weinberg RA. The Hallmarks of Cancer. Cell. 2000;100(1):57-70. doi: 10.1016/s00928674(00)81683-9. PubMed PMID: 10647931.

16. Dunn GP, Old LJ, Schreiber RD. The three Es of cancer immunoediting. Annu Rev Immunol. 2004;22:329-60.

17. Movahedi A, Mostajaboddavati M, Rajabibazl M, Mirfakhraie R, Enferadi M. Association of telomere length with chronic exposure to ionizing radiation among inhabitants of natural high background radiation areas of Ramsar, Iran. International Journal of Radiation Biology. 2019;95(8):1113-21. doi: 10.1080/09553002.2019.1605460.

18. Das B, Saini D, Seshadri M. Telomere length in human adults and high level natural background radiation. PloS One. 2009;4(12):e8440. doi:10.1371/ journal.pone.0008440.

19. Bevelacqua JJ, Welsh J, Mortazavi SMJ. Comments on "Association of telomere length with chronic exposure to ionizing radiation among inhabitants of natural high background radiation areas of Ramsar, Iran". International Journal of Radiation Biology. 2020;96(6):1-2. doi: 10.1080/09553002.2020.1739770. 
20. Bevelacqua JJ. Health physics in the 21st century. John Wiley \& Sons; 2008.

21. Muñoz-Lorente MA, Cano-Martin AC, Blasco MA. Mice with hyper-long telomeres show less metabolic aging and longer lifespans. Nature Communications. 2019;10(1):1-4. doi: 10.1038/s41467019-12664-X.

22. Mortazavi SMJ, Bevelacqua JJ, Welsh J. Comments on "The NASA Twins Study: A multidimensional analysis of a year-long human spaceflight". Science. 2019.

23. Welsh J, Bevelacqua JJ, Keshavarz M, Mortazavi SAR, Mortazavi SMJ. Is Telomere Length a Biomarker of Adaptive Response? Controversial Findings of NASA and Residents of High Background Radiation Areas. J Biomed Phys Eng. 2019;9(3):381-8. doi: 10.31661/JBPE.V9|3JUN.1151. PubMed PMID: 31341884. PubMed PMCID: PMC6613149.
24. Bevelacqua JJ, Mortazavi SMJ. Comments on "IS telomere shortening reversible? A clue from NASA's twins mission”. Polymorphism. 2019;3:1-3.

25. Arbeev KG, Verhulst S, Steenstrup T, Kark JD, Bagley 0 , et al. Association of Leukocyte Telomere Length With Mortality Among Adult Participants in 3 Longitudinal Studies. JAMA Network Open. 2020;3(2):e200023. doi: 10.1001/jamanetworkopen.2020.0023. PubMed PMID: 32101305. PubMed PMCID: PMC7137690.

26. Halaschek-Wiener J, Khattra JS, McKay S, Pouzyrev A, et al. Analysis of long-lived C. elegans daf-2 mutants using serial analysis of gene expression. Genome Research. 2005;15(5):603-15. doi: 10.1101/gr.3274805.

27. Zullo JM, Drake D, Aron L, O'Hern P, Dhamne SC, Davidsohn N, et al. Regulation of lifespan by neural excitation and REST. Nature. 2019;574(7778):35964. doi: 10.1038/s41586-019-1647-8. 